\title{
Starting early: integration of self-management support into an acute stroke service
}

Petra Mäkelä, Sara Gawned, Fiona Jones

St George's Healthcare NHS Trust, and Kingston University and St George's University of London

\begin{abstract}
Self-management support following stroke is rare, despite emerging evidence for impact on patient outcomes. The promotion of a common approach to self-management support across a stroke pathway requires collaboration between professionals. To date, the feasibility of selfmanagement support in acute stroke settings has not been evaluated.
\end{abstract}

The Bridges stroke self-management package (SMP) is based on self-efficacy principles. It is delivered by professionals and supported by a patient-held workbook. The aim of this project was to introduce the Bridges stroke SMP to the multidisciplinary staff of a London hyperacute and acute stroke unit.

The 'Plan Do Study Act' (PDSA) cycle guided iterative stages of project development, with normalisation process theory helping to embed the intervention into existing ways of working. Questionnaires explored attitudes, beliefs and experiences of the staff who were integrating selfmanagement support into ways of working in the acute stroke setting.

Self-management support training was delivered to a total of 46 multidisciplinary stroke staff. Of the staff who attended the follow-up training, $66 \%$ had implemented Bridges self-management support with patients since initial training, and $100 \%$ felt their practice had changed. Questionnaire findings demonstrated that staff attitudes and beliefs had changed following training, particularly regarding ownership and type of rehabilitation goals set, and prioritisation of self-management support within acute stroke care.

Staff initiated an audit of washing and dressing practices pre- and post-training. This was designed to evaluate the number of occasions when techniques were used by staff to facilitate patients' independence and self-management. They found that the number of occasions featuring optimum practice went from $54 \%$ at baseline to $63 \%$ at three months post-training.

This project demonstrated the feasibility of integrating self-management support into an acute stroke setting. Further work is required to evaluate sustainability of the Bridges stroke SMP, to understand the barriers and opportunities involved in engaging all professional groups in integrated self-management support in acute stroke settings, and to assess patient reported outcomes.

\section{Problem}

Recent decades have seen significant developments in the management of stroke as an acute event, particularly with the implementation of thrombolysis [Chiu et al, 1998; Sandercock et al, 2012; Wardlaw et al, 2014] and increasing interest in endovascular therapy [Ciccone et al, 2013]. The role of organised stroke unit care is well established in significantly improving outcomes after acute stroke [Govan et al, 2008]. However, the focus on the acute stroke episode is disproportionate in relation to unmet needs as identified by patients themselves, [McKevitt et al, 2010; 2011; CQC 2011] including the recognised 'abandonment' that patients feel after leaving hospital [National Audit Office, 2005].

Alongside the intense medical care, diagnostic testing, and therapeutic interventions that characterise the acute inpatient stage following stroke, individuals face evolving personal challenges. Although strategies to cope with the consequences of a stroke may improve in the longer term [King, 1996; Pound et al, 1998; Woodman et al, 2014], unfolding events and interactions can affect beliefs about personal capabilities and these may influence subsequent behaviour [Strecher et al, 1986; Ellis-Hill et al, 2009; Jones, 2010].

Admissions for stroke have increased in London since the London stroke model was introduced in 2010, with reduced lengths of stay on acute stroke units (ASU). Many patients are discharged home directly from the hyperactute stroke unit (HASU) within 72 hours [Hunter et al, 2013]. The short lengths of stay and highly medicalised environment have not been considered appropriate to introduce patients to the principles of self-management (e.g. develop personal goals and action plans, be supported in problem solving, share expertise, and make decisions). However, staff on an acute stroke unit could be in a unique position to introduce patients to the principles of self-management. They could provide greater continuity with approaches used in post-hospital rehabilitation stroke services, or could provide support to those who do not receive further rehabilitative input following their discharge home.

The evidence base for self-management programmes delivered to 
the stroke population is growing [Lennon et al, 2013]. The UK national clinical guideline for stroke recommends: "All patients should be offered training in self-management skills, to include active problem-solving and individual goal setting" [4th Edition, 2012, p.93], and "active support for self-management" has been considered to be a top priority for commissioners [Naylor et al, 2013, p.3]. However, the concept of self-management support encompasses highly heterogeneous interventions. To date, the focus has predominantly been on interventions centred on the patient [Kennedy et al, 2007a], with less attention to "the way in which clinicians' skills and attitudes can be changed to enable them to support patients in their efforts to self-manage" [Newbronner et al, 2013, p.6].

Although self-management support is now being introduced into rehabilitation and care post-stroke [Jones and Riazi, 2011], to our knowledge this has not yet been attempted within the acute inpatient stroke setting.

\section{Background}

Self-management support intends to facilitate behaviour change, influence an individual's ability to cope with their condition, and enhance quality of life [de Silva, 2011]. It is distinct from patient education or skills training [Barlow et al, 2002; Jones and Riaizi, 2011] and from interventions to increase compliance with recommended treatments [Walker et al, 2003].

The Bridges stroke self-management package (SMP) is an intervention delivered to individuals by professionals, and supported by a patient held workbook [Jones et al, 2009; McKenna et al, 2014]. Bridges stroke SMP is based on self-efficacy principles, defined as "the belief in one's capabilities to organize and execute the courses of action required to produce given attainments" [Bandura, 1997]. An overview of training and implementation components of Bridges stroke SMP is shown in Figure 1 (see attachment).

Bridges promotes a whole systems approach to self-management, considering individual patient needs, professional views and organisational issues. Bridges stroke SMP has been introduced to stroke pathways that include some acute stroke unit staff [Jones and Bailey, 2013] but has not previously been introduced to an acute stroke unit as a whole.

Challenges have previously been demonstrated in the translation of research findings on self-management support into clinical contexts [Kennedy et al, 2013]. Of note, inadequate consideration of the "relevant attitudes and possibly skills" of healthcare professionals has been considered to be a contributory factor when wholesystems approaches fail [Sun and Guyatt, 2013, p.1]. Divergent views on the overall value of an initiative can present a significant barrier to implementation [Lloyd et al, 2013].

Stroke teams comprise a variety of professional backgrounds, and practices such as goal setting, a key component of selfmanagement support, can subtly differ. Although the stroke rehabilitation guidance from NICE states that patients should be provided with the "support they need to make decisions and take an active part in setting goals" [NICE 2013, p.25], research suggests that clinicians perceive this way of working to be more time consuming, and that processes involve clinicians' prioritisation of certain goals over others [Levack et al, 2011]. Furthermore, professionals may hold beliefs and assumptions about an individual's readiness for self-management [Jones, 2006], and may perceive difficulties in its introduction in the early stages poststroke, for example in the context of patients with cognitive and communication problems.

The aim of this project was to integrate the Bridges stroke SMP to the multidisciplinary team working in a London HASU and ASU, and in doing so to explore the attitudes and beliefs held about selfmanagement support in this context.

\section{Baseline measurement}

The Bridges stroke SMP training involved two workshops for stroke unit staff, spanning four months. The measurement strategy aimed to capture the impact of this training on multidisciplinary staff members' attitudes and beliefs, and to inform the future measurement of impact on team processes and patient experience. Data were collected in the following ways, over a total of a six month period:

1. Questionnaires were administered to staff members immediately prior to the training, at an interim stage between the first and second training workshops, and following completion of training. The questionnaire items were designed to assess attitudes, beliefs, and knowledge about self-management support, and were modified to the acute stroke context from a questionnaire used within similar project across a stroke pathway [Jones and Bailey, 2013].

2. Questionnaires were developed and piloted with patients to gauge their perceptions of how involved they felt in their rehabilitation and care, as well as their experiences of goal setting, problem solving, and decision making. These methods will inform ongoing work in the area.

3. Feedback questionnaires were used directly after the workshops for evaluation of the quality of training delivered to staff members.

4. Baseline data were captured on the washing and dressing practices on the acute stroke unit, to enable an assessment of the impact of Bridges training on everyday interdisciplinary rehabilitation processes that can encourage and promote self-management.

See supplementary file: ds4061.pdf - "Figure 1: Overview of Bridges Stroke Self-Management Package"

\section{Design}

To date, Bridges stroke SMP has been developed, implemented, and assessed primarily within post-acute, rehabilitation stroke settings [Jones and Bailey, 2013]. As the focus within this project 
BMJ Quality Improvement Reports

was on implementation within a hyperacute and acute stroke service, the key features of the project design related to the challenges involved in its introduction to the multidisciplinary team's ways of working, within this new context.

The approach to integration into the acute stroke team's ways of working was informed by normalisation process theory [NPT; May et al, 2009]. This provides a framework through which to assess facilitators and barriers to the incorporation of complex interventions into routine practice, to the point of becoming 'normalised' within the setting [Murray et al, 2010]. NPT comprises four key components that interact with each other and with the organisational culture and processes. These are illustrated below, through examples of their application within this project:

Coherence:

- Illustrating the difference between Bridges SMP and established staff interactions with patients, such as the local goal-setting approach

- Explanation of underpinning Social Cognitive Theory [Bandura, 1997].

Cognitive participation:

- Accommodating professionals' shared and differing beliefs

- Methods for incorporation of Bridges SMP into ways of working

- Facilitating engagement through flexible training slots, availability of material such as patient workbooks, staff peer mentoring.

Collective action:

- Consideration, discussion and action planning for specific demands of the acute stroke setting

- Integration into current service processes: team meetings, goal-setting, documentation, handover provided on discharge.

Reflexive monitoring:

- Staff and patient feedback questionnaires

- Individual case reflections after implementation of Bridges SMP training

- Online staff discussion forum

- Local 'champions' to motivate, embed, sustain and evolve the approach

\section{Strategy}

The strategy implemented iterative PDSA cycles encompassing the four components of NPT outlined above, with the aim of normalising Bridges self-management support in the acute stroke setting. The resulting multilevel approach intended to facilitate sustainable change, taking into account the barriers and facilitators specific to the context.
PDSA Cycle 1:

The aim of the initial cycle was to explore specific challenges and opportunities relating to using Bridges in the acute stroke setting. Discussions with the multi-professional project team identified possible difficulties in the feasibility of staff on shift work accessing the training (mainly nurses and health care assistants). The training timetable was developed to include shorter sessions and options over a series of days, which was found to facilitate attendance of those staff groups. Other professional groups (speech and language therapy, and physicians) were not represented within the attendees and their inclusion will require further consideration.

PDSA Cycle 2:

In order to encourage attendance at training, and facilitate coherence and cognitive participation (as described above), activities were undertaken to raise awareness about the initiative. Presentations were delivered to a multi-professional group, flyers were circulated, and posters were displayed in prominent staff locations. Email reminders were also distributed, pre-training reading was sent to team leaders, and dissemination was encouraged. Following delivery of training, flyers were modified to include a list of Bridges catch-phrases that staff could use to include a stronger focus on self-management within interactions with patients.

PDSA Cycle 3:

The aim of this cycle was to ensure the content of the Bridges stroke SMP training would be relevant to the acute stroke setting, to enable collective action by the staff and patients. This involved modification of the existing Bridges stroke SMP content to include a greater emphasis on interactions to support self-management, and to develop shared understanding of processes for supporting selfmanagement between different professional groups. In addition, a questionnaire was developed and piloted with patients to explore ways to evaluate their experiences of involvement in their rehabilitation, and their views on opportunities for self-management.

PDSA Cycle 4:

The aim of this cycle was to deliver the training workshops and to assess any associated changes in attitudes, beliefs, and their prior knowledge about self-management support. This was collected through questionnaires both between and following the two half-day workshops. The collection of interim questionnaires (around two months after the initial training) was hindered by insufficient computer access for staff to enable online completion, and limited numbers of hard copies were returned at this time point. However, case reflections were collected on how practice had changed, with examples of how self-management had been supported in the acute stroke unit.

PDSA Cycle 5:

This cycle aimed to develop strategies and team processes to integrate Bridges stroke self-management principles into everyday 
BMJ Quality Improvement Reports

clinical practice in the acute stroke setting. This drew upon reflexive monitoring of experiences of staff, throughout the training and thereafter. A network of Bridges champions held meetings to determine on their own action plans. Staff were interviewed about their experiences for a feature article within the bi-annual Bridges newsletter, disseminated to stroke teams and practitioners around the UK.

PDSA Cycle 6:

Consideration was given to the sustainability of Bridges stroke selfmanagement within the service. For those staff members who had not attended the training, or required a refresher session, further inservice training was led by the Bridges champions. Discussions included the issue of the key professional groups who had not attended the training, and the potential impact on collective action within an integrated team approach. An additional workshop was delivered for the medical staff but, during this project, it was not possible to engage speech and language therapists in the training.

\section{Results}

Staff questionnaires:

Training was delivered to a total of 46 staff. Completed questionnaires were collected from 30 staff members prior to the training, 14 at the interim stage and 37 following the training. Figure 2 shows the breakdown of professional groups who attended training (see attachment).

Around one third had been working in stroke for more than five years. Approximately $50 \%$ of each group were working with patients from day one after admission with stroke, and a further $40 \%$ of each group were working with people in the first month after stroke. Approximately $80 \%$ were working in the acute stroke unit setting, with a further $40 \%$ also working on HASU.

The percentage who endorsed a statement that the acute stroke unit was "not the right setting" for self-management support remained low throughout: $7 \%$ prior to training and at the interim stage, and $8 \%$ post-training.

Following Bridges training, changes were demonstrated in the attitudes and beliefs of staff in relation to issues integral to selfmanagement support. Percentages of staff agreeing or strongly agreeing with key statements included in the questionnaires are shown in Table 1 (see attachment). Of note, the percentage endorsing a statement "the clinician should lead goal-setting" reduced from $59 \%$ pre-training to $28 \%$ at the interim stage, and $21 \%$ post-training. An example of change in attitudes relating to collaborative goal-setting related to the statement "unrealistic goals hold back progress", for which the percentage in agreement reduced from $54 \%$ pre- to $14 \%$ post-training.

Of the staff who attended the follow-up training, $66 \%$ reported that they had implemented Bridges stroke self-management support within their work, and $71 \%$ felt there was "enough time for introducing self-management". In addition, $100 \%$ felt their practice had changed and would recommend the training to others.

Patient questionnaires:

The response rate from patient questionnaires was low, in part because of the relatively fast through-put, and attempting to capture feedback just before discharge. Those available demonstrated a range of responses regarding the extent to which patients felt involved with their rehabilitation and care, and their experiences of being supported to self-manage. These preliminary findings will inform further development of data capture tools, which can be integrated into pre-discharge planning.

Impact on interdisciplinary processes:

Washing and dressing practices were assessed, exemplifying everyday processes on the acute stroke unit. As the degree to which a person could participate in their washing and dressing varied, an 'ideal standard' was described for each individual in terms of optimal incorporation of self-management support, informed by an occupational therapy wash and dress assessment. Data were collected for one month, at baseline and also three months after completion of Bridges training (data collected on weekdays only). The individually determined washing and dressing 'ideals' were compared with observation of the approach actually taken to patients' washing and dressing, as supported by the nursing staff. Reasons for deviation from the individual 'ideals' were captured and categorised. Frequencies of observations are shown in Table 2 (see attachment).

Although the sample sizes at each time point differed, this preliminary assessment of impact of Bridges multidisciplinary training suggests an increase in the percentage of occasions when staff implemented the ideal approach to washing and dressing, facilitating patients' independence, and self-management. There was a reduction in the percentage of occasions where lack of staff knowledge was deemed to be the predominant reason that prevented the ideal approach to self-management from occurring.

See supplementary file: ds4062.pdf - "Results: Figure 2, Table 1, Table 2"

\section{Lessons and limitations}

Stroke training in the UK is often discipline-specific. We learned that, for self-management support to be integrated successfully into stroke unit activities and processes, it requires the whole team to be trained together in order to reach shared understanding of what it is, and how it can be operationalised and sustained over time. This is strongly supported in self-management research relating to programmes used outside of stroke [Kennedy, 2007b].

Following staff training, several positive changes occurred that helped to facilitate a shared approach to self-management support, including the development of the role of the Bridges champions and a multi-disciplinary project group to monitor and spread good practice. A number of changes were also put into place to enable a greater role for patients in their self-care, thus promoting self- 
management. This included occupational therapists and nurses working together to redesign how patients were supported to wash and dress, to enable patients to have choice and control over when they were washed and given time to practice on their own before being supported by nursing staff.

Several lessons were learned particularly in relation to carrying out the project. Not all professional groups will engage with training, but this does not necessarily mean that they do not support this way of working. Medical staff were unable to attend, but a shortened session was delivered to address the key principles of the Bridges stroke SMP and illustrate ways in which medical staff could support self-management through their own interactions with colleagues and patients. Some staff did have initial concerns about using a selfmanagement approach with patients with mood and cognitive issues, but these were largely addressed through the training and modelling by champions that showed alternative ways that interactions can support self-management, as well as how to involve family members.

We recognised from patient feedback that most perceived their stroke consultant to be 'in charge', and that any interactions with the doctor can have a powerful impact on factors which contribute directly to self-management such as hope, anticipated prognosis, and involvement in planning for the future. Key principles from the Bridges stroke SMP include facilitating problem solving, goal setting, self-discovery and reflection on progress by patients recovering from stroke, in order that they can gain confidence and practice the skills required for self-management. The role of medical staff in facilitating these skills in patients should not be underestimated, and we would advocate medical staff being supported to attend the whole training package with their teams in future programmes. Lack of engagement from influential clinicians in attending self-management training, changing their own practice, and supporting the new system has been demonstrated to undermine implementation, especially through a negative effect on junior staff [Newbronner et al, 2013]. In view of these implications, we have developed and reported on findings from a national survey, to explore opportunities and barriers for the involvement of physicians in supporting self-management after stroke [Mäkelä et al, 2014].

As part of a whole systems approach to self-management, we recognised a greater-than-anticipated need for the organisational perspectives (such as multidisciplinary meeting agendas, assessment schedules, and goal setting meetings) to adapt, in order to enable self-management to remain a prominent part of all aspects of service delivery. These are critically important in order to fully integrate self-management into everyday work practices. There is also a need to simultaneously address patient-reported barriers [Joseph-Williams et al, 2013]. This learning related specifically to the collective action required for the normalisation of any new way of working, as described by Murray and colleagues [2010].

\section{Conclusion}

This project has demonstrated that it is feasible to integrate selfmanagement support into an acute stroke setting. Staff changed their attitudes regarding ownership and type of goals set, and recognised the need to prioritise self-management support within acute stroke care. Further work is required to evaluate the sustainability of integrated self-management support in acute stroke settings, as well as to consider how to engage all professional groups in training and delivery. We have demonstrated that it is possible to enable good uptake from nursing staff and health care assistants in multidisciplinary training, providing enough time and care is spent to adapt the delivery of the training.

Real changes in collaborative ward processes are required in order to integrate self-management support into an acute stroke setting, and while there were examples of practice change among nursing staff, occupational therapists and physiotherapists, further work is required to find ways of attracting and involving medics and speech and language therapists fully in the Bridges SMP training. This is vital in order to reach a shared understanding not just about selfmanagement support, but also how to operationalise greater involvement of patients in their own care.

\section{References}

Bandura A. The nature and structure of self-efficacy. In: Selfefficacy: the exercise of control. Bandura A, ed. New York: W.H Freeman and Company, 1997

Barlow J, Sturt J and Hearnshaw H. Self-management interventions for people with chronic conditions in primary care: examples from arthritis, asthma and diabetes. Health Educ J 2002;61:365-78

Bodenheimer, T, Lorig, K, Holman, H \& Grumbach, K. Patient selfmanagement of chronic disease in primary care. J Am Med Assoc 2002; 288(19):2469-75

Care Quality Commission. Supporting life after stroke: A review of services for people who have had a stroke and their carers. London: Care Quality Commission, 2011

Chiu D, Krieger D, Villar-Cordova C, Kasner SE, Morgenstern LB, Bratina PL, et al. Intravenous tissue plasminogen activator for acute ischemic stroke feasibility, safety, and efficacy in the first year of clinical practice. Stroke 1998;29(1):18-22

Ciccone A, Valvassori L, Nichelatti M, Sgoifo A, Ponzio M., Sterzi R, et al. Endovascular treatment for acute ischemic stroke. $N$ Engl J Med 2013;368(10):904-13

De Silva D. Helping people help themselves: A review of the evidence considering whether it is worthwhile to support selfmanagement. London: The Health Foundation, 2011

Ellis-Hill C, Robison J, Wiles R, McPherson KM, Hyndman D \& Ashburn A. "Going home to get on with life": Patients and carers experiences of being discharged from hospital following a stroke. Disabil Rehabil 2009;31(2):61-72

Govan L, Weir CJ, Langhorne P, for the Stroke Unit Trialists C. Organized inpatient (stroke unit) care for stroke. Stroke 
Harris R, Sims S, Hewitt G, Joy M, Brearley S, Cloud G, et al. Interprofessional teamwork across stroke care pathways: outcomes and patient and carer experience. Final report. NIHR Service Delivery and Organisation programme; 2013 [Accessed 18.05.14] http://www.nets.nihr.ac.uk/ data/assets/pdf file/0005/85091/FR-08 -1819-219.pdf

Hunter RM, Davie C, Rudd A, Thompson A, Walker H, Thomson N, et al. Impact on clinical and cost outcomes of a centralized approach to acute stroke care in London: a comparative effectiveness before and after model. PloS ONE 2013;8(8):e70420

Jones F, Mandy A \& Partridge C. Changing self-efficacy in individuals following first stroke: preliminary study of a novel selfmanagement intervention. Clin Rehabil 2009;23(6):522-33

Jones F and Riazi A. Self-efficacy and self-management after stroke: a systematic review. Disabil Rehabil 2011;33(10):797-810

Jones F and Bailey N. How can we train stroke practitioners about patient self-management? Description and evaluation of a pathway wide training programme. Eur J Pers Cent Health, 2013;1(1):246-54

Joseph-Williams N, Elwyn G, \& Edwards A. Knowledge is not power for patients: A systematic review and thematic synthesis of patientreported barriers and facilitators to shared decision making. Pat Ed Couns 2014;94(3):291-309

Kennedy A, Reeves D, Bower P, Lee V, Middleton E, Richardson $G$, et al. The effectiveness and cost effectiveness of a national lay led self care support programme for patients with long-term conditions: a pragmatic randomised controlled trial. J Epidemiol Comm Health 2007a;61:254-61

Kennedy A, Rogers A, et al. Support for self care for patients with chronic disease. BMJ 2007b;335:968-70

Kennedy A, Bower P, Reeves D, Blakeman T, Boweb R, ChewGraham $\mathrm{C}$, et al. Implementation of self management support for long term conditions in routine primary care settings: cluster randomised controlled trial. BMJ 2013;346:f2882

King R. Quality of life after stroke. Stroke 1996;27:1467-72

Lennon S, McKenna S, \& Jones F. Self-management programmes for people post stroke: a systematic review. Clin Rehabil 2013;27(10):867-78

Levack WM, Dean SG, Siegert RJ, \& McPherson KM. Navigating patient-centered goal setting in inpatient stroke rehabilitation: how clinicians control the process to meet perceived professional responsibilities. Pat Ed Couns 2011;85(2):206-13

Lloyd A, Joseph-Williams N, Edwards A, Rix A, \& Elwyn G. Patchy 'coherence': using normalization process theory to evaluate a multifaceted shared decision making implementation program (MAGIC).
Mäkelä P, Abdul Saheb M, Bhalla A, Moynihan B and Jones F. A survey of physicians' views of self-management support after acute stroke: obstacles and opportunities. Cerebrovasc Dis 2014;37(suppl 1):1-2;309 (DOI:10.1159/000362854)

May CR, Mair F, Finch T, MacFarlane A, Dowrick C, Treweek S, et al. Development of a theory of implementation and integration: Normalization Process Theory. Implement Sci 2009;4:29

McKenna, S, Jones F, Glenfield P and Lennon S. Bridges selfmanagement programme for people with stroke in the community: feasibility randomised controlled trial. Int J Stroke, 2013 [Accessed 11.01.14] http://onlinelibrary.wiley.com/doi/10.1111/ijs.12195/abstract

McKevitt C, Fudge N, Redfern J, Sheldenkar A, Crichton S, \& Wolfe C. The Stroke Association UK Stroke Survivor Needs Survey: Final Report. London, Stroke Association, 2010

McKevitt C, Fudge N, Redfern J, Sheldenkar A, Crichton S \& Rudd AR. Self-reported long-term needs after stroke. Stroke 2011;42(5):1398-403

Murray E, Treweek S, Pope C, MacFarlane A, Ballini L, Dowrick C. Normalisation process theory: a framework for developing, evaluating and implementing complex interventions. BMC Med 2010;8:63 [Accessed 11.01.14] http://www.biomedcentral.com/1741-7015/8/63

National Audit Office. Faster access to better stroke care. 2005 [Accessed 11.01.14] http://collection.europarchive.org/tna/20060731065549/http://www.n ao.org.uk/publications/nao_reports/05-06/0506452es.pdf

National Institute for Health and Care Excellence. CG162 Stroke rehabilitation: full guideline, 2013 [Accessed 18.05.14] http://guidance.nice.org.uk/CG162/Guidance/pdf/English

Naylor C, Imison C, Addicott R, Buck D, Goodwin N, Harrison T, et al. Transforming our health care system: ten priorities for commissioners London: King's Fund, 2013 [Accessed 11.01.14] http://www.kingsfund.org.uk/sites/files/kf/field/field_publication_file/tr ansforming-health-care-system-ten-priorities-commissionersmay11.pdf

Newbronner L, Chamberlain R, Borthwick M, Baxter M \& Sanderson D. Sustaining and spreading self-management support: Lessons from Co-creating Health Phase 2. London: Health Foundation, 2013

Pound P, Gompertz P, Ebrahim S. A patient-centred study of the consequences of stroke. Clin Rehabil 1998;12:255-64

Royal College of Physicians Intercollegiate Working Party for Stroke. National Clinical Guidelines for Stroke (Fourth Edition). London, 2012 


\section{BMJ Quality Improvement Reports}

Sandercock P, Wardlaw JM, Lindley RI, Dennis M, Cohen G, Murray $G$, et al. The benefits and harms of intravenous thrombolysis with recombinant tissue plasminogen activator within 6 $\mathrm{h}$ of acute ischaemic stroke (the third international stroke trial [IST-3]): a randomised controlled trial. Lancet 2012;379(9834):2352-63

Strecher VJ, Mcevoy B, Becker MH, Rosenstock IM. The role of selfefficacy in achieving health behaviour change. Health Educ Behav 1986;13(1):73-92

Sun $X$ and Guyatt $G$. Interventions to enhance self management support. BMJ 2013;346:f3949

Walker C, Swerissen $\mathrm{H}$, Belfrage J. Self-management: its place in the management of chronic illness. Australian Health Review 2003;26(2):34-42

Wardlaw JM, Murray V, Berge E \& del Zoppo GJ. Thrombolysis for acute ischaemic stroke. Cochrane Database Syst Rev 2014;7.CD000213. DOI: 10.1002/14651858.CD000213.pub3

Woodman, P, A. Riazi, Pereira C \& Jones F. Social participation post stroke: a meta-ethnographic review of the experiences and views of community-dwelling stroke survivors. Disabil Rehabil 2014;(0):1-13

\section{Declaration of interests}

We have read and understood BMJ policy on declaration of interests and declare the following interests: Fiona Jones is the Founder of Bridges stroke self-management and Director of Bridges self-management. The other authors have nothing to declare.

\section{Acknowledgements}

We would like to acknowledge the help and support of the stroke unit champions team for their input: Jessica Styles, Hannah Brooke, Emma Kelly, Mark Sheridan and Iwona Haponik. 